\title{
LA RESPONSABILIDAD SOCIAL EMPRESARIAL. REVISIÓN PARA EL SECTOR SOLIDARIO
}

\section{CORPORATE SOCIAL RESPONSIBILITY. REVISIÓN FOR THE SOLIDARITY SECTOR}

\author{
Msc (C). José Andrés Bayona Soto ${ }^{\mathrm{a}}$, Msc. Genny Torcoroma Navarro Claro ${ }^{\mathrm{b}}$, Ph.D. Sir Alexci \\ Suarez Castrillón ${ }^{\mathrm{c}}$
}

${ }^{a}$ Universidad Francisco de Paula Santander Ocaña, Grupo de Investigación GIDSE, Vía Acolsure Sede el Algodonal, Ocaña - Norte de Santander, Colombia, jabayonas@ufpsp.edu.co

${ }^{\mathrm{b}}$ Universidad Francisco de Paula Santander Ocaña, Grupo de Investigación GIDSE, Vía Acolsure Sede el Algodonal, Ocaña - Norte de Santander, Colombia, gtnavarroc@ufpso.edu.co

${ }^{\mathrm{c}}$ Universidad Francisco de Paula Santander Ocaña, Grupo de Investigación GRUCITE, Vía Acolsure Sede el Algodonal, Ocaña - Norte de Santander, Colombia, sasuarezc@ufpso.edu.co

Resumen: El sector solidario, economía social o economía solidaria, se caracteriza por emprendimientos de iniciativa social que desde su esencia de trabajo, están alineados con los postulados de la responsabilidad social empresarial (RSE). La intención de éste artículo es presentar los resultados de la revisión del tema de la responsabilidad social empresarial en el sector solidario. El documento se divide en tres partes realizando un abordaje teórico, conceptual y analítico. En la primera parte se demuestran postulados teóricos relacionados con la premisa de los principios de la economía solidaria como fundamento de la responsabilidad social empresarial en organizaciones del sector solidario. En la segunda parte se realiza un compendio teórico y analítico demostrando la concepción de los gobiernos corporativos como sistema de dirección y control para el aseguramiento de prácticas adecuadas de responsabilidad social en dichas organizaciones. Por último se realiza un acercamiento teórico, conceptual y analítico hacia la sostenibilidad y desarrollo sostenible como un direccionamiento estratégico que es apoyado por la responsabilidad social empresarial en las organizaciones del sector solidario. Para finalizar se expresarán algunas conclusiones particulares acerca del tema.

Palabras clave: Responsabilidad social empresarial, sector solidario, principios de la economía solidaria, Gobiernos corporativos, Sostenibilidad. 


\begin{abstract}
The solidary sector, social economy or solidary economy is characterized by social initiative initiatives that, from the point of view of their work, are aligned with the principles of corporate social responsibility (CSR). The purpose of this article is to present the results of the review of the topic of corporate social responsibility in the solidarity sector. The document is divided into three parts, making a theoretical, conceptual and analytical approach. The first part demonstrates theoretical postulates related to the premise of the principles of solidarity economy as the foundation of corporate social responsibility in organizations of the solidarity sector. In the second part, a theoretical and analytical compendium is made demonstrating the conception of corporate governments as a system of direction and control for the assurance of adequate practices of social responsibility in said organizations. Finally, a theoretical, conceptual and analytical approach towards sustainability and sustainable development is carried out as a strategic direction that is supported by corporate social responsibility in the organizations of the solidarity sector. Finally, some specific conclusions about the subject will be expressed.
\end{abstract}

Keywords: Corporate Social Responsibility, Solidarity Sector, Principles of Solidarity Economy, Corporate Governance, Sustainability.

\section{INTRODUCCIÓN}

Las organizaciones pertenecientes al sector solidario, fundamentan su razón de ser en los principios y valores de la economía solidaria y su carácter no lucrativo; a su vez se caracterizan por la búsqueda de beneficios para sus asociados y las comunidades. Es así como el sector se conforma y se construye a partir de emprendimientos sociales representados por las organizaciones solidarias de desarrollo (Corporaciones, Fundaciones) y las organizaciones de la economía solidaria (Cooperativas, Precooperativas, Fondos de empleados, Asociaciones mutuales); quienes mediante el desempeño de sus actividades socioeconómicas enmarcan su desempeño en los principios de la solidaridad y la cooperación, y bajo la premisa de la integralidad de su gestión; demostrando de ésta forma una identificación clara con los estamentos de la responsabilidad social empresarial, proceso consciente y voluntario que tiene como finalidad la construcción de confianza con los grupos de interés (stakeholders) mediante el desarrollo de una gestión completa que involucre la interrelación consecuente entre los aspectos económicos, sociales y medioambientales.

El desarrollo del presente artículo tiene como propósito, hacer una aproximación reflexiva al tema de la responsabilidad social empresarial, asumiendo un enfoque aplicado al sector solidario; intentando reconocer las implicaciones que la responsabilidad social empresarial establece en determinación de los principios y valores del sector solidario, la formación de capacidades de Gobierno Corporativo para la dirección y control de los procesos de responsabilidad social empresarial, y por último brindar una visión de acercamiento hacia la sostenibilidad y el desarrollo sostenible de las organizaciones pertenecientes al tercer sector, economía solidaria, economía social; denominaciones como también se reconoce al sector solidario.

El trabajo de revisión adelantado, no es de ninguna manera una corriente de 
pensamiento definitiva del proceso de la responsabilidad social empresarial para las organizaciones del sector solidario; la información presentada reconoce la razonabilidad del tema en ocasión de las condiciones y circunstancias dadas por los medios y los contextos derivados de los entornos socioeconómicos específicos y cambiantes; así mismo se tiene como referencia los tamaños estructurales (En cuanto a su número de trabajadores y niveles de capital) y la complejidad administrativa de las organizaciones. Por supuesto la globalización y su incidencia inminente, los problemas de la sociedad en todos los ámbitos, ubica al tercer sector como modelo de entendimiento y participación, tal como lo expresa (Pradini \& Sánchez, 2007) motivando la intencionalidad de involucrar y empoderar una responsabilidad compartida para hacer frente a las necesidades $y$ exigencias que enfrenta una sociedad global, responsabilidad fundada en la cooperación y solidaridad e identificada con el concepto y práctica de la responsabilidad social. A partir de lo anteriormente expuesto es consciente afirmar la necesidad intrínseca de realizar una continua y constante reflexión al respecto, con el ánimo de brindar oportunidad y capacidad a las organizaciones del sector solidario, para que en cabeza de sus estructuras de Gobierno Corporativo, se direccionen estratégicamente hacia la sostenibilidad y el desarrollo sostenible, en apoyo y correspondencia a las prácticas suficientes y adecuadas de responsabilidad social empresarial.

\section{METODOLOGÍA}

En cuanto a lo metodológico se aplicó un trabajo de integración de perspectivas, relacionados con la discusión de la responsabilidad social empresarial para el sector solidario; ajustando de manera razonable la relevancia temporal de artículos de investigación relacionados con el tema, realizando un procedimiento de selección de estos mediante una estructura de clasificación coherente con la intención del proceso de revisión. De esta manera se adelantó el proceso de revisión, que redundó en una idea de ilación bajo subcategorías identificadas, previo reconocimiento generalizado de información en bases de datos y revistas indexadas. La idea consistió en buscar la esencia del tema, y configurar variables que respondieran a preguntas tales como ¿Por qué? ¿Cómo? Y ¿Para qué? En el estudio de revisión de la responsabilidad social empresarial en el sector solidario, intentando de manera congruente una aproximación reflexiva en cuanto a lo conceptual y teórico del tema y nuestro abordaje particular del mismo.

\section{DESARROLLO DE LA REVISIÓN DEL TEMA.}

LOS PRINCIPIOS Y VALORES DE LA ECONOMÍA SOLIDARIA; COMO UNA PREMISA QUE FUNDAMENTA LAS PRÁCTICAS DE RESPONSABILIDAD SOCIAL EN LAS ORGANIZACIONES DEL SECTOR SOLIDARIO.

La economía solidaria, del tercer sector, economía social o economía de las sociedades cooperativas se caracteriza por emprendimientos de iniciativa social que desde su esencia de trabajo, están alineadas con la responsabilidad social empresarial (RSE); (Sánchez \& Vaca, 2005) afirman que las sociedades cooperativas se acomodan a los planteamientos de la RSE a partir de su sistema de creencias, aplicados en el marco de los principios y valores que fundamentan 
su funcionamiento, teniendo principal atención como elemento diferenciador, la atención y la búsqueda constante de un equilibrio con sus grupos de interés (stakeholders), inspirador de confianza.

La generación de confianza es una premisa fundamental de la RSE asumida según (Belhouari, Buendía, Lapointe, \& Tremblay, 2005) como un valor estratégico empresarial que redunda en la integralidad económica, social y medioambiental; en ajuste y determinación de los grupos de interés y por supuesto de los valores y dinámicas que identifican a las sociedades cooperativas.

Teniendo en cuenta que el tercer sector es un emprendimiento social que propende por el bienestar de las sociedades en diferentes ámbitos a partir de una ocupación específica, en concordancia con los preceptos de la economía solidaria; Castro (2006), citado por (Marcuello, 2007) generaliza que para las entidades integrantes de la economía social se promueve un modelo basado en la solidaridad, cohesión, equidad e inserción social muy cercano a lo que se puede denominar "Responsabilidad social".

En cuanto a la ocupación (Marcuello, 2007) afirma que estos emprendimientos (Empresas no lucrativas) desarrollan dos funciones: 1) Productoras de bienes y servicios, y 2) gestoras de recursos públicos; dejando claro que estas dos funciones en la realidad identifican una gran diversidad y complejidad, debido a factores tales como: El tamaño de la organización, la naturaleza jurídica adoptada, las actividades socioeconómicas y las relaciones con diversos grupos de interés.
Las organizaciones del sector solidario, deben reflexionar continuamente sobre el sentido y propósito de la responsabilidad social corporativa, comprendida como lo determina un informe de la Pricewaterhouse Coopers en 2003: La responsabilidad social empresarial es una inversión a largo plazo que afecta positivamente a la rentabilidad de la empresa, al mejorar la reputación, la obtención de ventajas competitivas, y las relaciones con los stakeholders.

Una organización perteneciente a la economía solidaria, cualquiera que sea su actividad surge de un emprendimiento social que con base a un capital gestionado bajo las pautas de la economía solidaria; tiene por objeto generar una confianza interna $\mathrm{y}$ externa, que no es más que la creación de valor social y económico para comprender una visión de crecimiento en conjunto con los grupos de interés, que se fundamenta en el ejercicio de un trabajo reflexivo que parte de las motivaciones construidas sobre quienes laboran en la organización, procurando mejorar las condiciones de las personas de una comunidad, bajo el reconocimiento idóneo de derechos, libertades y capacidades humanas (Barrera, 2007).

Las necesidades y exigencias que buscan satisfacer cualquier tipo de organización del tercer sector parten de los problemas sociales, políticos, ambientales, culturales y económicos entre otros, que no garantizan el bienestar de las comunidades. (Cortés \& Belmonte, 2010), reconocen que desde la concepción de estas entidades demuestran y han velado por comportamientos socialmente responsables bajo el fomento, desarrollo y orientación de sus asociados en las dinámicas de la economía social. A su 
vez (Cortés \& Belmonte, 2010) ubican una posición reflexiva al incorporar una discusión sobre el riesgo de la efectiva implantación de la RSE debido a la posibilidad de aparición de una mercantilización de su actividad que tergiversa la actividad como ente social, determinando menor participación de los asociados y trasgrediendo la RSE y su alineación con los valores y principios cooperativos, tal como lo contempla (Mozas \& Puentes, 2010).

Por su parte contrarrestar y evitar el riesgo de perder el direccionamiento dado por los principios y valores corporativos en una organización de la economía solidaria, inhibe en esencia su posibilidad de éxito empresarial, desvirtuando y desnaturalizando su razón de ser (Pedrosa \& Hernández, 2011).

En razón de ello, (Sánchez \& Gallardo, 2013) nos permiten inferir en el estudio de este criterio de la investigación; que el énfasis de las organizaciones del tercer sector, está garantizado por el cumplimiento consciente de los principios de la economía solidaria y/o cooperativismo, como una premisa que fundamenta el diseño organizacional y las sus prácticas de responsabilidad social

\section{ESTRATEGIAS DE GOBIERNO CORPORATIVO; COMO DESARROLLO DEL ASEGURAMIENTO DE PRÁCTICAS ADECUADAS DE RESPONSABILIDAD SOCIAL EN ORGANIZACIONES DEL SECTOR SOLIDARIO.}

Las directivas de una empresa bien sea de carácter lucrativo o no lucrativo; donde dentro de estas últimas mencionadas se encuentran clasificadas las organizaciones pertenecientes a la economía solidaria, se establece la necesidad de mostrar resultados que demuestren la responsabilidad social empresarial RSE. Un resultado que contribuye a tales efectos es el denominado: Balance social cooperativo, la cual es una herramienta de gestión a partir de una contabilidad que se enfoca en los objetivos y se aplica mediante la utilización de indicadores sociales que demuestran una realidad más allá de las cifras económicas (Mugarra, Responsabilidad y balance social hoy en día: un reto para las cooperativas, 2001). A su vez (Moreno, 2004) en consonancia a ello demuestra la importancia y la influencia que la implementación de la RSE genera en la empresas en su estabilidad, cohesión económica, competitividad empresarial, reconociendo un plazo de mediano y largo plazo que tiene por objetivo la retroalimentación constante con los grupos de interés, para forjar asertividad en la satisfacción y más que ello la generación de valor.

Las organizaciones no lucrativas, como toda empresa; a parte de su naturaleza jurídica y actividad económica ejercida, también son el reflejo de su comportamiento a partir de la gestión. La RSE en las organizaciones, es producto de una gestión integrada que confluyen sobre la ejecución de procesos económicos, sociales y medioambientales que se encaminan a la elaboración, presentación y seguimiento de los correspondientes informes de sostenibilidad, como producto de su gestión integrada (Server \& Villalonga, La responsabilidad social corporativa (RSC) y su gestión integrada, 2005).

Contextualizando en las estructuras organizacionales de las empresas del sector solidario y su direccionamiento identificado con la concepción debida como gobiernos corporativos; (Belhouari, Buendía, Lapointe, 
\& Tremblay, 2005) establecen que la gestión dentro de éstas deben partir del hecho consciente de adoptar como valor estratégico la integración congruente de los aspectos económicos, sociales y medioambientales como un funcionamiento idóneo que generan impactos importantes sobre la rentabilidad y la competitividad, sirviendo de influencia positiva y en doble vía de las relaciones con sus grupos de interés.

La economía solidaria como fuerza emergente de generación de empresa, reconocida por primar la persona por encima del capital, teniendo por objeto sumar utilidades cuidadas hacia el beneficio compartido buscando entre otros aspectos que se relacionan con RSE: la generación de empleo, el fomento y creación de un capital social, la regulación y equilibrio de sectores económicos y sociales, desarrollo de espíritu emprendedor, activación de la participación en la gestión económica, formación de habilidades para la gestión empresarial, inclusión e inserción social y laboral de colectivos de difícil empleabilidad, integración de personas con discapacidad a la actividad económica, acceso a la vivienda, servicios educativos, creación de infraestructuras y dotaciones sociales, aglutinación de trabajo autónomo, asistencia sanitaria personalizada, grupos empresariales con distribución de riqueza equitativos (De Castro, 2005).

Como lo afirma (García \& Pedrosa, 2011) la búsqueda de un gobierno corporativo para una organización cooperativa o del tercer sector es realizar actos que busquen la consecución del desarrollo y la cohesión social en la zona de influencia de la organización, identificando dentro de este contexto, las practicas éticas, la atención a la satisfacción de los grupos de interés y el manejo importante de una cultura que permita un desenvolvimiento propicio $\mathrm{y}$ adecuado en cuanto al desarrollo sostenible de la comunidad.

El trasfondo de la RSE va más allá de la presentación de los informes aunque en estos se demuestren los índices de favorabilidad e impactos que se derivan de las actividades de una organización en el marco de las relaciones con su medio y su contexto. La medición de los impactos generados por la actividad empresarial, siempre ha sido una apuesta para forjar la razonabilidad y la concientización que contribuyan al logro de los objetivos que sumergen a las organizaciones en prácticas de RSE, donde cabe destacar que el componente normativo y de regulación, así como la forma de realizar dichas mediciones deberían establecer un nivel de normalización que permita orientar los cometidos sociales, económicos y medioambientales tanto en su ejecución como en su evaluación. (Yenni, Cardona, \& Rendón, 2013).

Los escenarios de incertidumbre moral dentro de la economía y el comportamiento ético poco reflexionado por parte de los directivos de las organizaciones, influencian las estructuras de gobierno corporativo en alineación con la transparencia que demuestre la información de la organización. Tal como se viene desarrollando el abordaje teórico del cometido de la investigación la transparencia confluye con la solidaridad y la sostenibilidad de las empresas pertenecientes a la economía solidaria, observando las premisas dadas por los 
sistemas de principios y valores por los que se deberían identificar tales empresas, y esto con especial atención con los informes económicos, los cuales deben tener una mirada social con ponderación de objetivos e impactos, tal como lo afirma (Socías, 1999) citado por (Socias \& Horrach, 2013):es obvio que la información estrictamente económica no representa el rendimiento del proceso producto en entidades no lucrativas. (Socias \& Horrach, 2013) Conllevan el sentido de la transparencia como un medio de gran reconocimiento que permite el posicionamiento en un sector, considerando una fuente imborrable de credibilidad $y$ legitimidad.

Es por ello que según (Muñoz, Laborda, \& Briones, 2013) las organizaciones de la economía solidaria requieren de gestión, y por consiguiente de gobiernos corporativos que se conviertan en organismos con un enfoque de gestión que según la premisa de la RSE inmerso por supuesto en el contexto económico, social y medioambiental estas organizaciones se transformes y se construyan en una versión de oportunidad y capacidad con carácter innovador en pro del mejor desarrollo de las comunidades, incentivado dichas prácticas de gobierno corporativo en el marco de políticas tales como la integración social.

Los gobiernos corporativos de las organizaciones serán los únicos que direccionen, reglamenten una adecuada y suficiente RSE dentro de las empresas; y en mayor medida en las organizaciones de economía solidaria, en el marco de los principios y valores corporativos que las definen $y$ las orientan, optando y desarrollando practicas adecuadas que permitan la verdadera implementación y por ende el aseguramiento de su correcta aplicación y oportuno seguimiento. En el contexto de la globalización y los intereses de las organizaciones de la economía solidaria según (Serna \& Rubio, 2016) quienes tienen la responsabilidad de direccionarlas a emprender e implantar estrategias cuyos resultados conduzcan al logro de los objetivos planeados, deben buscar obtener ventajas comparativas, conquistar propósitos a partir la incorporación y ejecución de mejores prácticas de gobierno, redundando en el beneficio de los grupos de interés y generando valor y una mejor gestión de los riesgos.

\section{SOSTENIBILIDAD Y DESARROLLO SOSTENIBLE; COMO DIRECCIONAMIENTO ESTRATÉGICO, APOYADO POR UN PROCESO DE RESPONSABILIDAD SOCIAL EN LAS ORGANIZACIONES DEL SETOR SOLIDARIO.}

Bajo las tendencias de cumplimiento social, económico y medio ambiental que las organizaciones deben observar; la identificación con la responsabilidad social empresarial (RSE) es funcional, propicia y de amplia estudio y reflexión constante para adecuar prácticas de gobierno corporativo que redunden en oportunidad y capacidad para el desempeño idóneo de sus objetivos empresariales. Así pues (Andreu, 2005) nos muestra que la responsabilidad social empresarial es algo que más amplio que la acción social y la filantropía; y que el alcance de la RSE tiene que ver en mayor proporción por la forma de gestionar, más que una función reconocida en el organigrama. 
Es así como (Garzón, Amaya, \& Castellanos, 2004) establecen que a partir del vertiginoso avance de la información, las comunicaciones, el conocimiento y la tecnología han transformado las dinámicas en todos los ámbitos, conllevando a que las organizaciones tengan que enfrentarse a dichas condiciones y circunstancias para tener la capacidad de adaptarse y mantenerse en el tiempo, es decir concebir la sostenibilidad y el desarrollo sostenible.

La RSE y desarrollo sostenible son conceptos de valor que están a la vanguardia e innovación social que no son exclusivamente diseñadas para las grandes empresas de capital, sino que deben ser adaptadas a la especificidades de las cooperativas (Mugarra, Memoria de sostenibilidad: una propuesta adaptada para las cooperativas de enseñanza en el país Vasco, 2005).

La RSE para empresas de economía solidaria, grandes o pequeñas según su tamaño y volumen de operaciones; deben partir del hecho de incorporación del concepto de solidaridad en su misión social, por lo que se infiere que dicha misión estará direccionada en el marco de la sostenibilidad, bajo la manifestación de la transparencia como fundamento que dimensione de manera justa y adecuada lo que es su propósito integral.

En consecuencia el desarrollo sostenible en el momento de decidir voluntariamente implementar conscientemente la RSE implica la comprensión de sus enfoques conceptuales, por parte de quienes dirigen las organizaciones y tener en cuenta la concepción, medición y evaluación de indicadores ajustados a ello, con implicación del desarrollo humano y la integralidad social, económica y medioambiental (Arias, 2006).

Así mismo su implantación, conduce a un nuevo modelo de empresa que amplía su preocupación hacia una nueva dimensión empresarial, que se puede sintetizar en el concepto de sostenibilidad, que abarca a un mismo nivel aspectos económicos, sociales y medioambientales. La responsabilidad social empresarial (RSE) conduce a un modelo de empresa no basada exclusivamente en satisfacer la rentabilidad de sus socios o accionistas, sino que adopta otros compromisos relacionados con la sociedad (Server \& Villalonga, La responsabilidad social en el cooperativismo de crédito. El fondo de educación y promoción como indicador para su evaluación.Estudio empírico para le caso español., 2007)

El tercer sector de la economía y la actividad económica de ahorro y crédito, deben ser entendidas como organizaciones con un alto grado de interacción con la Sociedad (Dávila, 2004) citado por (Sánchez \& Rendón, 2008). Así pues la sostenibilidad de este tipo de organizaciones requiere de una comprensión de su naturaleza y su actividad en retroalimentación con los grupos de interés; única manera de considerar la RSE como un componente que vaya más allá de los principios y valores que identifican la economía solidaria.

De manera inevitable hay que reconocer que las organizaciones del denominado tercer sector nacen como respuesta a la desigualdad $y$ por consiguiente a la 
exclusión del sistema tradicional, iniciativas sociales adaptadas de manera idónea a los principios y valores de la economía solidaria, enfocando aspectos de todo un sistema, tales como la política. Estos espacios de iniciativa social se crean bajo una convicción, tal como lo expresan Roberto Ayala y Sergio Reuben en su artículo: Estado, globalización y política en América Latina, citado por (Quesada, 2008); donde se exponen políticas sociales que se dirigen a: 1) Reeducar a los pobres para que se vinculen al mercado, 2) Promover la autosuficiencia económica orientadas al impulso y apoyo de las microempresas, 3) Incentivar a las cooperativas para que asuman el riesgo de convertirse en empresas rentables y que puedan desplazar el viejo fundamento solidario, y 4) Las organizaciones sociales para que desarrollen negocios con los recursos de sus afiliados. Todo ello contribuye con el desarrollo sostenible.

Estructuras organizacionales como las cooperativas pertenecientes a la economía solidaria bien concebidas, trabajadas coherentemente de conformidad con los principios y valores cooperativos contribuyen al desarrollo sostenible, en la medida que la actividad socioeconómica se fundamenta en la utilización de recursos endógenos en la zona de influencia, funcionando responsables socialmente desde la integralidad de lo económico, social y medioambiental (Poyatos \& Velasco, 2009).

Es importante tener en cuenta que el panorama de las organizaciones de iniciativa social, pertenecientes a la economía solidaria se desarrolla en un ambiente de riesgos y la RSE se pone en tela de juicio debido a la no claridad e incertidumbre de la intención de las directivas de las organizaciones por diversas situaciones $\mathrm{y}$ variados aspectos. Este aspecto requiere regulación y reglamentación tal y como lo contempla (Server \& Capó, 2009) manifestando que el establecimiento de estas políticas orientadas al desarrollo de una gestión integrada de la $\mathrm{RS}$ va a generar en las empresas un efecto positivo en la obtención de ventajas competitivas incrementando su eficiencia así como su viabilidad a largo plazo. Igualmente reconocer según (Server \& Capó, 2009) que para lograr llevar a cabo la RSE es fundamental la interrelación entre los direccionamientos y prácticas de responsabilidad social de la organización, generando identidad con los grupos de interés, comprendiendo una gestión integrada.

Por su parte la identificación de los grupos de interés con la RSE de una organización es factor fundamental para comprobar la existencia de valor en dichas relaciones. En temas de economía a partir de actividades de carácter financiero, las organizaciones que se dedican a esta actividad deben tener especial cuidado en el mantenimiento de las relaciones con sus grupos de interés y la observancia debida y juiciosa de las expectativas de estos que permitan de esta manera involucrar estas premisas en las directrices de la responsabilidad social empresarial (Ruiz, De los Ríos, \& Tirado, 2009).

Es indispensable que toda organización socialmente responsable conciba un entendimiento de que los grupos de interés no son solo sus propietarios; ya que la perspectiva adecuada contempla una visión más amplia con otros grupos de interés 
como clientes, proveedores y la ciudadanía comunidad en general (Sajardo \& Serra, 2009).

La reflexión continua de la RSE como proceso de apoyo hacia el aseguramiento de la sostenibilidad de las organizaciones de la economía solidaria, debe estar en constante estudio ya que este tipo de economía, tal y como lo expresa (Pereira, 2009) constituye una realidad heterogénea compleja, marcada por dificultades y contradicciones en cuanto a la concepción, delimitación y mensuración.

Es así que la actuación de las organizaciones del tercer sector, debido a las implicaciones que generan sus comportamientos, debes ser organizaciones garantes de su esencia y su verdadera razón de ser, y tal y como lo expresa (Fajardo, 2009) poner de manifiesto su contribución al desarrollo económico y social y justificar a partir de lo adecuado de sus comportamientos políticas públicas que contribuyan a esta realidad con sujeción oportuna a las leyes, regulaciones y reglamentos existentes para los efectos de la actuación debida y coherente.

Una vez comprometido con el cumplimiento normativo; las organizaciones deben madurar en asertividad bajo la comprensión de lo que es sostenibilidad y desarrollo sostenible siendo esta la meta de toda organización, por lo que es primordial que se profundice en este cometido, puesto que tal y como nos lo comparte razonablemente (Saravia, 2010) no se puede concebir que ser sostenible es crecer como organización, ya que crecer no es un indicador oportuno de la buena gestión, factor fundamental para comprometerse con la sostenibilidad de toda empresa, bien sea lucrativa o no lucrativa.
Si no se reflexiona por parte de las organizaciones, hasta comprender que la sostenibilidad es el verdadero objetivo, se corre el riesgo de fallar en la gestión y los direccionamientos establecidos por los gobiernos corporativos de las organizaciones. (Maussa, 2010) nos comparte que la responsabilidad de quienes dirigen a las organizaciones es la autogestión, comprendida como el principio de conciencia, estableciendo que los modelos de gestión deben estar direccionados más allá de las características culturales, administrativas y de gestión por dicho principio.

Retomando lo concerniente a la actividad económica del ahorro y crédito; (Castro \& Romero, 2011) nos invita a contemplar la ética desde el concepto de banca ética, reconociendo ambientes de riesgos $y$ eventualidades de crisis, y llevando a cabo una idea oportuna de implementación a partir de la esencia de los principios cooperativos.

Como se ha dicho antes, la información de sostenibilidad producto del desempeño del objeto socioeconómico de las organizaciones de la economía solidaria, es el resultado de la forma en que se gestionan este tipo de organizaciones en atención a sus grupos de interés, reconociendo que la información cuando es transparente refleja el cumplimiento y logro de un requisito fundamental bajo la perspectiva de la responsabilidad social empresarial (Horrach \& Socias, 2011) 
A la luz de la comprensión reflexiva de todo el abordaje de RSE en organizaciones de economía solidaria; en retrospectiva y haciendo hincapié que al momento de generar emprendimientos de este tipo que sean sostenibles, se debe establecer un compromiso con el acercamiento al cumplimiento del objeto social, desarrollar la capacidad de arraigarse a un territorio concreto, operar con un proceso de encadenamiento de empresas de un mismo tipo y en especial el fomento de procesos autogestionados y participativos (Arboleda \& Zabala, 2011)

La autogestión como principio, la RSE como propósito, el gobierno corporativo como estamento, los grupos de interés como misión y la sostenibilidad como objetivo de las organizaciones sin ánimo de lucro pertenecientes al sector de la economía solidaria; requieren de una sinergia constante entre ética, responsabilidad social y reputación, a partir de la progresión de programas y actividades tal como nos lo indican (Olmedo, Martínez, Arcas, \& Longinos, 2012).

Al continuar el proceso de comprensión de la sostenibilidad como objetivo de toda empresa, es inminente saber que el sector de la economía solidaria como componente de un sistema socio económico, genera influencia y por lo tanto impactos sobre el ámbito económico y social en general. Los avances de la economía solidaria en el territorio colombiano es de principal atención tal como nos lo indica (Limas \& Higuera, 2012).

Insistiendo con la temática (Poveda, 2013) concluye que el desarrollo sostenible ha sido una acumulación de estrategias desde lo lógico e histórico: Cumplimiento de la legislación, producción limpia, ecoeficiencia, RSE y economía verde.

Los informes de sostenibilidad son el modo de divulgación para evidenciar los comportamientos de la RSE; así pues (Bollas, Seguí, \& Polo, 2014) ya que la sostenibilidad en los últimos tiempos es un aspecto de relevancia en el mundo empresarial y la tendencia genera voluntariedad consciente para ser demostrada para la difusión y retroalimentación con los grupos de interés.

La sostenibilidad involucra la gestión de los riesgos desarrollada por las directivas de la organización, comprendidos los riesgos como aquellos eventos con probabilidad de ocurrencia que generan consecuencias. Esto es tomado en cuenta por (Patón, 2014) estableciendo que debe existir un control de los riesgos sociales que son evidenciados oportunamente con las prácticas de RSE. Al administrar los riesgos conscientemente las organizaciones direccionan un marco efectivo de responsabilidad social.

El gobierno corporativo como estamento de la gestión estratégica de una organización representada por el gerente, y teniendo como criterio esta responsabilidad y competencia y teniendo en cuenta el Pacto global en Colombia, según (Duque, Ortíz, \& Arciniegas, 2014) se estableció una caracterización de este perfil, encontrando que este cargo se presenta de carácter emergente. 
Volviendo al caso de la organización social como un proceso gestado por un emprendimiento social, La sociedad genera iniciativas sociales innovadoras y con fortalezas suficientes para alcanzar el éxito, pero el camino para su desarrollo y sostenibilidad está repleto de dificultades, tanto por el planteamiento del modelo de negocio y su posterior gestión como por la escasa sensibilidad y vinculación de las instituciones públicas y entidades financieras a este tipo de proyectos (Vásquez \& Abeal, 2015)

\section{CONCLUSIONES.}

Es propio e interés de este artículo, sentar un posicionamiento a partir de una revisión teórica y conceptual desde un enfoque reflexivo y analítico; que buscó reconocer el por qué el sector solidario mantiene una caracterización ejemplar para los propósitos de la responsabilidad social empresarial, dejando claro que es misión de las organizaciones pertenecientes a este sector de la economía, ser garantes de confianza dentro del entorno social y económico, desde el fundamento otorgado por los principios y valores que se incorporan desde su existencia y desde el desempeño activo, participativo e interviniente que asumen en beneficio de sus asociados y comunidades, determinando así una estrecha relación entre los principios y valores solidarios con los preceptos de la responsabilidad social empresarial.

La responsabilidad social empresarial es un compromiso, que debe ser concebido por un sistema de dirección y control en cualquier organización. Los direccionamientos de Gobiernos corporativos de las organizaciones solidarias deberían ser estructuras robustas que contribuyan al crecimiento y desarrollo, y que lo hagan siguiendo un incesante espíritu de reflexión hacia la perfectibilidad, declarando $\mathrm{y}$ gestionando un funcionamiento cada vez más solidario y más cooperativo, incorporando la responsabilidad social como un proceso con esencia y con forma, estableciendo de esta manera una perspectiva de cómo hacerlo.

La sostenibilidad y el desarrollo sostenible son un ideal que se debe buscar a partir de la adecuada y suficiente administración de recursos y la debida y ajustada concepción de propósitos. Las organizaciones pertenecientes al sector solidario deben mejorarse a sí mismas para luego intentar mejorar condiciones y circunstancias en la sociedad, velando por criterios de importante significación para bien de su responsabilidad; es decir para su responsabilidad social empresarial.

\section{REFERENCIAS}

Andreu, A. (2005). La responsablidad social corporativa: un concepto por definir. CIRIEC.

Arboleda, O., \& Zabala, H. (2011). Condiciones clave para el éxito y sostenibilidad de los emprendimientos solidarios en Medellín. Semestre Económico.

Arias, F. (2006). Desarrollo sostenible y sus indicadores. Revista sociedad y economía.

Barrera, E. (2007). La empresa social y su responsabilidad social. Innovar.

Belhouari, A., Buendía, I., Lapointe, M., \& Tremblay, B. (2005). La responsbailidad social de las empresas: ¿un nuevo valor para las cooperativas? CIRIEC. 
Bollas, H., Seguí, E., \& Polo, F. (2014). Informes de sostenibilidad en cooperativas de crédito: un análisis de su divulgación en Europa. Revesco.

Castro , M., \& Romero, N. (2011). Cooperativas de crédito y banca ética ¿Un camino por explorar? CIRIEC.

Cortés, F. J., \& Belmonte, L. J. (2010). La base social de las cooperativas de crédito. la importancia de la responsabilidad social corporativa. Revista de estudios empresariales.

De Castro, M. (2005). La responsabilidad social de las emrpesas, o un nuevo concepto de empresa. CIRIEC.

Duque, Y., Ortíz, M., \& Arciniegas, J. (2014). Perfiles de los gerentes de responsabilidad social de las organizaciones adheridas al pacto global en colombia. Investigación y desarrollo.

Fajardo, G. (2009). La economía social en las leyes. CIRIEC.

García, E., \& Pedrosa, C. (2011). El gobierno en la sociedad cooperativa como base de la cohesión social: el caso de una sociedad cooperativa agraria. Revesco.

Garzón, D., Amaya, C., \& Castellanos, O. (2004). Modelo conceptual e instrumental de sostenibilidad organizacional a partir de la evaluación del tejido social empresarial. Innovar.

Horrach, P., \& Socias, A. (2011). La actiud de las empresas de economía solidaria frente a la divulgación de información sobre sostenibilidad desde le prisma de la teoría de los stakeholders o grupos de interés. Revista de contabilidad.
Limas, S., \& Higuera, J. (2009). Impacto socieconómico del sector de la economía solidaria en el departamento de Boyaca, Colombia. 2000 - 2009. Apuntes del CENES.

Limas, S., \& Higuera, J. (2012). Impacto socieoeconómico del sector de la economía solidaria en el departamento Boyaca Colombia. 2000 - 2009. Apuntes del CENES.

Marcuello, C. (2007). Responsabilidad social y organizaciones no lucrativas.

Ekonomiaz.

Marcuello, C. (2007). Responsabilidad social y organizaciones no lucrativas.

Ekonomiaz.

Maussa, F. (2010). Modelo alternativo para la sostenibildad empresarial. Cuadernos de administración.

Moreno, J. Á. (2004). Responsbilidad social coprporativa y competitividad: Una visión desde la empresa. $B B V A$.

Mozas, A., \& Puentes, R. (2010). La responsabilidad social corporativa y su paralelismo con las sociedades cooperativas. Revesco.

Mugarra, A. (2001). Responsabilidad y balance social hoy en día: un reto para las cooperativas. CIRIEC.

Mugarra, A. (2005). Memoria de sostenibilidad: una propuesta adaptada para las cooperativas de enseñanza del país vasco. CIRIEC.

Muñoz, M., Laborda, F., \& Briones, A. J. (2013). Gestión económica para el buen gobierno corporativo en las entidades de la economia social. Tourism \& Management studies. 
Olmedo, I., Martínez, I., Arcas, N., \& Longinos, J. (2012). Relación circular entre ética, responsabilidad social y reputacción de las cooperativas. Revesco.

Patón, G. (2014). La fiscalidad de las cooperativas desde la perspectiva de la internacionalización de la economía social y el desarrollo sostenible.

Derecho PUCP.

Pedrosa, C., \& García, E. (s.f.).

Pedrosa, C., \& Hernández, M. J. (2011). ¿Cómo aplican las sociedades cooperativas de éxito los principios cooperativos? El caso del Grupo Hojiblanca. CIRIEC.

Pereira, L. (2009). Economía social y economía solidaria en Brasil: Consideraciones conceptuales y prácticas en pro de la construcción de alternativas socioeconómicas al desarrollo territorial. Revista Anfora.

Poveda, I. (2013). El desarrollo sostenible a nivel empresarial. Ciencia en su PC.

Poyatos, R., \& Velasco, M. (2009). Importancia de las sociedades cooperativas como medio paa contribuir al desarrollo económico, social, y medioambiental, de forma sostenible y responsable.

Revesco.

Pradini, J., \& Sánchez, E. (2007). La responsbailidad social en el tercer sector. Salud y drogas.

Quesada, A. (2008). El tercer sector, la economía social y trabajo social en costa rica. Revista de ciencias sociales universidad de Costa Rica.

Ruiz, M., De los Ríos, A., \& Tirado, P. (2009). la resposnabilidad social y la crisis económica. ¿Responden las entidades financieras españolas a los grupos de interés? CIRIEC.

Sajardo, A., \& Serra, I. (2009). Impacto económico y social de la responsabilidad social empresarial en la comunidad valenciana. CIRIEC.

Sánchez, A., \& Vaca, R. M. (2005). Responsbailidad scoial corporativa y cooperativismo; vinculos y potencialidades. CIRIEC.

Sánchez, M. I., \& Gallardo, D. (2013). El papel del tercer sector ante la responsabilidad social empresarial. Un análisis cualitativo de la reallidad extremeña. CIRIEC.

Sánchez, X., \& Rendón, B. (2008). Craracterización del sector cooperativo de ahooro y crédito en el valle del cauca. Entramado.

Saravia, J. (2010). El problema del crecimiento sostenible ¿cómo crecer sin destruirse? Revista EAN.

Serna, H., \& Rubio, G. (2016). La gobernabilidad en el sector cooperativo: Una reflexión acerca de su verdadera implementación. Revista virtual Universidad católica del Norte.

Server, R., \& Capó, J. (2009). La responsabilidad social empresarial en un contexto de crisis. Repercucuón en las sociedades cooperativas. CIRIEC.

Server, R., \& Villalonga, I. (2005). La responsabilidad social corporativa (RSC) y su gestión integrada. CIRIEC.

Server, R., \& Villalonga, I. (2007). La responsabilidad social en el cooperativismo de crédito. El fondo de educación y promoción como indicador 
para su evaluación.Estudio empírico

para le caso español. Interciencia.

Socias, A., \& Horrach, P. (2013). Enfoque de la responsabilidad social y la transparencia en empresas de economía solidaria. CIRIEC.

S Vásquez, J., \& Abeal, J. (2015). Principales determinantes para la sostenibilidad a largo plazo de una iniciativa social emprendedora. Trabajo.

Yenni, D., Cardona, M., \& Rendón, J. (2013). Responsabilidad social empresarial: teorías, índices, estándares y certificaciones. Cuadernos de administración. 